\section{CHARACTERISTICS OF PATIENTS SURVIVING MORE THAN TEN YEARS AFTER CARDIAC TRANSPLANTATION}

The clinical status and quality of life of $\mathbf{4 0}$ patients who lived or are still alive more than 10 years after transplantation at our institution were reviewed with the use of our transplant database, prospective patient examinations, cardiac catheterization, and exercise testing. Patient-perceived health status was determined with use of the Nottingham Health Profile and General Well Being examinations. Factors associated with longevity were determined by a Cox proportional hazards model. Twentysix patients are alive and 14 have died. The mean age at transplant was $32.4 \pm 12$ years and the current age (or age at death) is $46.1 \pm 12.8$ years. Actuarial freedom from rejection was similar to that of patients surviving less than 10 years $(p=0.8)$, but freedom from all types of infection was less $(p=0.005)$. Immunosuppressive drugs include cyclosporine (11/26 patients), azathioprine (24/26), and prednisone (26/26, mean dose $12.7 \mathrm{mg} /$ day). Catheterization hemodynamic data show wellpreserved graft function at a mean follow-up of $11.7 \pm 3.3$ years. Graft coronary artery disease prevalence is $\mathbf{5 1 . 0 \%} \pm \mathbf{8 \%}$. Exercise test results are as follows: duration $8.7 \pm 3.5$ minutes (range 2 to 16 minutes), maximum heart rate/expected rate $77.3 \% \pm 11 \%(50 \%$ to $92 \%$ ), maximum systolic blood pressure $171 \pm 23 \mathrm{~mm} \mathrm{Hg}$ (140 to $208 \mathrm{~mm} \mathrm{Hg}$ ), and metabolic equivalents $9.2 \pm 2.3$ units (5.5 to 12.9 units), or about $84 \%$ of predicted. Mean score on the General Well Being examination was $75.3 \pm 21.6$ (normal). Nottingham Health Profile scores were nearly normal, except for in the 50- to 64-year-old age group in categories of mobility, pain, sleep quality, and energy level. Causes of death were coronary artery disease in 7 of 14, infection in 4 of 14, lymphoma in 1 of 14, and nonlymphoid cancer in 2 of 14. In the Cox regression, variables most associated with survival $(t>2.0$, multivariate $p=0.0005)$ were age at transplantation $(t=3.26)$, preoperative duration of illness $(t=3.57)$, postoperative cytomegalovirus infection $(t=2.16)$, and ejection fraction at 12 months after operation $(t=-2.62)$. We conclude that cardiac transplantation can provide patients with end-stage cardiac failure an acceptable general medical condition, functional status, and perceived quality of life well into the second decade after operation. (J THORAC CARDIOVASC SURG 1995;109:1103-15)

William M. DeCampli, MD, PhD, Helen Luikart, MS, RN, Sharon Hunt, MD, FACC, and Edward B. Stinson, MD, Stanford, Calif.
C ardiac transplantation has proved to be a reasonable therapeutic option for selected patients with end-stage heart failure. This conclusion is supported by the favorable survival rate and postoperative quality of life of patients who have undergone cardiac transplantation, compared with these find-

From the Department of Cardiothoracic Surgery, Stanford University Medical Center, Stanford, Calif.

Read at the Twentieth Annual Meeting of The Western Thoracic Surgical Association, Olympic Valley, Calif., June 22-25, 1994.

Address for reprints: William M. DeCampli, MD, PhD, Division of Cardiothoracic Surgery, Children's Hospital Oakland, 74752nd St., Oakland, CA 94609.

Copyright (C) 1995 by Mosby-Year Book, Inc.

$0022-5223 / 95 \$ 3.00+0 \quad \mathbf{1 2 / 6 / 6 2 9 4 5}$ ings in those cases managed medically. ${ }^{1}$ Most studies that support this conclusion, however, have had mean or median follow-up periods of only a few years and thus do not provide evidence of the clinical status and perceived quality of life in the longer run, that is, beyond a decade after operation. This limitation is imposed by (1) the relatively low 10-year survival of patients operated on before 1982 (approximately $30 \%$ at Stanford) and (2) the existence of only a very few institutions that performed a significant number of transplantation operations before 1983. With more than 2000 heart transplant operations currently done per year in the United States, and an actuarial 10-year survival rate of approximately $50 \%$, an understanding of the clinical 
Table I. Dates of transplantation

\begin{tabular}{|c|c|c|c|c|c|}
\hline \multicolumn{3}{|c|}{ Living patients } & \multicolumn{3}{|c|}{ Deceased patients } \\
\hline Case No. & $\begin{array}{c}\text { Date of transplant } \\
\text { retransplant }\end{array}$ & $\begin{array}{c}\text { Current age } \\
(y r)\end{array}$ & Case No. & $\begin{array}{l}\text { Date of transplant; } \\
\text { retransplant }\end{array}$ & $\begin{array}{c}\text { Current age } \\
(y r)\end{array}$ \\
\hline 1 & $7 / 31 / 74 ; 6 / 30 / 86$ & 49 & 27 & $1 / 03 / 70$ & 63 \\
\hline 2 & $4 / 04 / 75$ & 45 & 28 & $11 / 08 / 71$ & 55 \\
\hline 3 & $4 / 22 / 76$ & 63 & 29 & $6 / 10 / 72$ & 65 \\
\hline 4 & $6 / 13 / 76$ & 67 & 30 & $5 / 11 / 74$ & 59 \\
\hline 5 & $11 / 08 / 76 ; 6 / 15 / 87$ & 40 & 31 & $8 / 19 / 74 ; 10 / 15 / 74 ; 10 / 13 / 85$ & 27 \\
\hline 6 & $1 / 25 / 77 ; 8 / 13 / 92$ & 30 & 32 & $8 / 25 / 74$ & 59 \\
\hline 7 & $2 / 08 / 77$ & 55 & 33 & $1 / 26 / 75 ; 02 / 15 / 81$ & 62 \\
\hline 8 & $5 / 28 / 77$ & 45 & 34 & $8 / 31 / 76$ & 48 \\
\hline 9 & $12 / 26 / 77 ; 8 / 10 / 89$ & 35 & 35 & $7 / 10 / 77$ & 60 \\
\hline 10 & $2 / 07 / 78$ & 56 & 36 & $11 / 03 / 77$ & 64 \\
\hline 11 & $4 / 23 / 78$ & 51 & 37 & $8 / 01 / 78$ & 53 \\
\hline 12 & $8 / 30 / 78$ & 35 & 38 & $2 / 13 / 80$ & 39 \\
\hline 13 & $1 / 20 / 79$ & 41 & 39 & $5 / 16 / 81$ & 30 \\
\hline 14 & $3 / 30 / 79$ & 50 & 40 & $7 / 29 / 81 ; 2 / 08 / 92$ & 28 \\
\hline 15 & $10 / 19 / 79$ & 34 & & & \\
\hline 16 & $11 / 18 / 79$ & 40 & & & \\
\hline 17 & $12 / 17 / 79$ & 26 & & & \\
\hline 18 & $12 / 21 / 79$ & 40 & & & \\
\hline 19 & $9 / 24 / 80$ & 42 & & & \\
\hline 20 & $11 / 23 / 80$ & 26 & & & \\
\hline 21 & $12 / 21 / 80$ & 30 & & & \\
\hline 22 & $1 / 13 / 81$ & 48 & & & \\
\hline 23 & $6 / 25 / 81$ & 53 & & & \\
\hline 24 & $6 / 30 / 81$ & 49 & & & \\
\hline 25 & $8 / 07 / 81$ & 24 & & & \\
\hline 26 & $10 / 04 / 81$ & 59 & & & \\
\hline
\end{tabular}

status and perceived quality of life more than a decade after operation is of obvious pertinence.

In this paper we present results of a study of 40 patients who are alive or who lived longer than 10 years after cardiac transplantation at a single institution. Specifically, we tested the following hypotheses about these long-term survivors: (1) the prevalence of graft coronary artery disease (CAD) continues to increase with time and graft $C A D$ is a significant source of mortality for this group of patients, (2) graft function is well-preserved beyond 10 years after transplantation, and (3) the exercise capacity of these patients and sense of well-being are reasonably close to those of the age-matched population at large.

Finally, we present a derivation of clinical factors that correlate with longer-term survival in our institution's total transplantation experience.

\section{Patients and methods}

The preoperative clinical status and postoperative clinical course (including regular follow-up history, physical examination, laboratory values, cardiac biopsy results, and cardiac catheterization data) of all patients undergoing heart transplantation at Stanford since 1970 were examined with the assistance of a database updated to January 1,1992 . The subset of patients who survived more than 10 years after their first heart transplant operation was examined in detail. Of the 661 patients in the database, 40 patients fell into this subset: 26 of these patients were alive and 14 had died. Seven of the 40 patients underwent cardiac retransplantation. All but two of these received retransplantation more than 10 years after the original operation. One patient had undergone retransplantation twice. The patients and their dates of transplantation are listed in Table $\mathbf{I}$.

The current clinical status of the living patients was further determined either at Stanford or by review of records of the patient's primary cardiologist. The evaluation included a review of active and past medical problems, current medications, family and social history (including smoking, drinking, and job or marital status change since transplantation), physical examination (including current weight), laboratory values (including hematocrit, blood urea nitrogen, creatinine, fasting glucose, total cholesterol, triglycerides), and data from all available cardiac catheterization reports. Graft CAD was assessed either angiographically or at autopsy. The incidence was 
determined only for CAD occurring in first-time allografts (that is, CAD in a second allograft was not considered). Actuarial freedom from CAD was calculated and compared with that in the set of all other patients who underwent transplantation at this institution. Infection and acute rejection data were obtained from the database. Presence of acute rejection was noted when a cardiac biopsy specimen showed International Society of Heart and Lung Transplantation grade II or higher.

So that functional status could be assessed, exercise tests were done on available patients. The actual exercise protocol varied for each patient; however, a "maximal" test was completed and maximum heart rate, blood pressure, duration, and exercise capacity (expressed in metabolic equivalents [METS] standardized for each exercise protocol) were determined. Results were compared with those of an age-matched sample of the normal population and with those of an age-matched cohort of patients who underwent transplantation an average of 7 months before evaluation.

So that we could evaluate patient-perceived health status or "sense of well-being," each patient in the subset completed both part I of the Nottingham Health Profile (NHP) and the General Well Being (GWB) examinations. The NHP examination is purported to evaluate "perceived distress." 2,3 The higher the score ( 0 to 100 for each of six sections), the more the distress. Weightings derived from the application by McKenna, Hunt, and McEwen ${ }^{4}$ of Thurstone's method of paired comparisons to the British population were applied to each question in the NHP survey. The GWB examination is best described as reflecting "expressions of psychiatric states of depression and anxiety or the absence thereof," according to Fazio. Scores from both tests were compared with those of published age and gender-matched norms. ${ }^{6}$ Correlations were calculated among the NHP and GWB scores, treadmill performance, employment and marital status, and number of current medical problems.

Because we were interested in factors that might contribute to (or at least be correlated with) the longevity of this subset of patients, two comparison groups were formed from the set of all patients who underwent transplantation at Stanford: (1) patients who survived 0.5 to 5.0 years after transplantation and (2) patients who survived 5 to 10 years after transplantation. The age at the time of transplantation; actuarial rates of freedom from acute rejection and from viral, bacterial, protozoal, and fungal infection; and cause of death were determined for each group and compared.

Finally, a formal multivariate analysis was done to further clarify the possible factors. Twenty-one variables were cited as possibly predictive of longevity. Variables evaluated at the time of transplantation included duration of preoperative illness, age at the time of transplantation, race, gender, hospital length of stay, number of human leukocyte antigen mismatches, serum creatinine level at the time of discharge, diastolic blood pressure at the time of discharge, donor age, donor race, donor gender, donor cytomegalovirus (CMV) status, and recipient CMV status. Variables evaluated at 1 year after operation included blood urea nitrogen, serum creatinine, diastolic blood pressure, left ventricular ejection fraction, degree of
CAD (quantitated by the number of vessels with significant stenoses), number of acute rejection episodes, number of episodes of infection, and incidences of CMV infection (as determined by the presence of inclusion bodies in tissue, positive culture, or greater than fourfold rise in anti-CMV immunoglobulin $\mathrm{G}$ serologic titer). Univariate analysis of variance was performed for each variable from the aforementioned three groups to obtain a qualitative estimate of significance. The nine most significant variables were determined. The set of all patients who underwent transplantation at this institution was then chosen and a subset determined that consisted of patients each meeting all of the following three criteria: (1) survival at least 1 year after operation, (2) not having retransplantation, and (3) having no missing data among the nine variables. By including only patients who survived at least 1 year, we eliminated operative and early postoperative complications and early refractory rejection and infection as causes of mortality. The final subset, consisting of 122 cases, was used in the multivariate analysis (see following section).

Results are expressed as mean plus or minus the standard deviation, unless otherwise specified. Comparison of means was made by one-way analysis of variance or Student's $t$ test. The Fisher exact test was used for nominal data in the analysis of the subset of patients receiving cyclosporine $(n=8)$, and the Mann-Whitney U test was used to compare variables between patients with and without evidence of CAD. Actuarial calculations were made with use of the product limit estimator with Greenwood's standard error. ${ }^{7}$ Comparison of actuarial curves was made by the Cox-Mantel test. Correlations were examined by determining Pearson coefficients or Spearman rank correlation coefficients. A value of $p<0.05$ was considered significant. The multivariate analysis was done by the stepwise proportional hazards method of Cox. ${ }^{8} \mathrm{~A}$ test of parallelism was made to ensure that the final covariates induced proportional (parallel) shifts of the arbitrary hazard function. A $\chi^{2}$ statistic was used to test the hypothesis that the gradient of the log-likelihood function was zero at its initial value, and $p<0.05$ was considered significant. The relative risk of death caused by an increase by $m$ units in a covariate was calculated as exp $(m b)$, where $b$ is the estimate of the normalized coefficient. Ninety-five percent confidence limits of the relative risk are given.

Henceforth the expression most recent refers to the most recently determined value of a variable in living patients or the last-determined value before death in deceased patients.

\section{Results}

Dates of transplantation and retransplantation for the group of patients surviving longer than 10 years are shown in Table I. The majority (32/40) underwent transplantation before the introduction of cyclosporine into our program in December 1980. Basic demographic data are listed in Table II. In comparison with patients in the short survival ( 0.5 to 5 years) group, the patients in the group with more 
Table II. Demographic characteristics*

\begin{tabular}{lcrl}
\multicolumn{1}{c}{ Item } & Mean & \multicolumn{1}{c}{ SD } & Range \\
\hline Age at transplant (yr) & 32.4 & 11.7 & $13-51$ \\
Current age (yr) & 46.1 & 12.8 & $24-67$ \\
Postop. years & 13.7 & 2.5 & $11-22$ \\
Admission weight (kg) & 65.8 & 11.4 & $43-92$ \\
Current weight $(\mathrm{kg})$ & 77.9 & 13.8 & $49-107$ \\
\hline
\end{tabular}

$S D$, Standard deviation.

*Sex: male, 35; female, 5.

than 10-year survival received transplantation at a younger mean age. In the former group, only 15 of 119 patients were younger than 30 years old at operation versus 18 of 40 for the long-term survivors $(p<0.001)$.

Actuarial freedom from acute rejection, in comparison with that of the two comparison groups, is plotted in Fig. 1. These curves are not significantly different and reflect the fact that by 0.5 years after operation only $15 \%$ of patients are free from at least one rejection episode. The mean number of infectious episodes was $2.24 \pm 2.1$. The actuarial freedom from such events, plotted for each of four major pathogen groups, is shown in Fig. 2. In each of the four pathogen categories, the long-term survivors had significantly greater freedom from infection compared with patients in the 0.5 - to 5-year $(p<$ $0.005)$ and 5- to 10 -year $(p<0.05)$ comparison groups. Similarly, when analyzed according to location of infection, the most significant difference favoring long-term survivors was found in the incidence of pneumonia. This fact is consistent with the fact that, when infection was the ultimate cause of death, the lungs were the most common primary site of infection.

The patients take an average of $6.6 \pm 2.7$ medications per day (range 3 to 14). All patients take prednisone at a mean dose of $12.7 \pm 3 \mathrm{mg} / \mathrm{day}$, and nearly all are taking azathioprine. Patients who underwent transplantation or retransplantation after December 1, 1980, are taking cyclosporine. Other common medications include a variety of antihypertensive drugs, diuretics, prophylactic antibiotics, and antiplatelet agents. Disorders possibly attributable to corticosteroid usage include hypertension $(55 \%)$, osteoporosis $(35 \%)$, cataracts (27\%), cushingoid appearance $(26 \%)$, diabetes mellitus $(24 \%)$, and weight gain $(19.8 \pm 17.8 \mathrm{~kg})$. Other chronic medical problems include seven cases of chronic renal insufficiency, 20 cases of graft CAD, and 27 noted miscellaneous problems (many attributable to adverse effects of medications).
Most recent laboratory values for the patients are shown in Table III. Of the eight patients with serum creatinine values higher than $1.8 \mathrm{mg} / \mathrm{dl}$, seven were taking cyclosporine and four of these seven had undergone retransplantation. These values are significantly higher than one would expect if the eight patients were a random subset of the group (Fisher's exact test, $p<0.01$ ). The mean total cholesterol and triglyceride values are influenced by one patient with isolated hypertriglyceridemia (most recent measurement $468 \mathrm{mg} / \mathrm{dl}$ ) and by two patients with combined disorders (cholesterol 445 and $293 \mathrm{mg} / \mathrm{dl}$, triglycerides 273 and $298 \mathrm{mg} / \mathrm{dl}$, respectively). For five patients evaluated in the retransplantation subgroup, values significantly higher than those in the first-time transplantation subgroup included blood urea nitrogen $(36.0 \pm 7.9 \mathrm{mg} / \mathrm{dl}, p=0.01)$, creatinine $(2.0 \pm 0.27 \mathrm{mg} / \mathrm{dl}, p=0.002)$, and total cholesterol $(257.2 \pm 33.7 \mathrm{mg} / \mathrm{dl}, p=0.02)$.

The most recent cardiac catheterization data, obtained at a mean of $11.7 \pm 3.3$ years after operation, are displayed in Table IV. A remarkable feature is the relatively normal hemodynamic data, indicating, on average, well-preserved graft function at this long follow-up interval. Aortic diastolic pressure and left ventricular end-diastolic pressure were mildly elevated. The prevalence of $\mathrm{CAD}$ was $51.0 \% \pm 8.0 \%$ (standard error of the estimate) at a mean of $11.7 \pm 3.3$ years of angiographic or autopsy follow-up. For each patient, we determined the earliest postoperative time in which angiographic evidence of graft $C A D$ was detected: this mean value was $9.3 \pm 4.1$ years $(n=40)$. The actuarial freedom from CAD for the study group is shown in Fig. 3 together with that for all other patients who underwent transplantation at Stanford. These two curves are not statistically significantly different. Values for number of acute rejection episodes, aortic diastolic pressure, serum cholesterol, current age, current weight, left ventricular end-diastolic pressure, and NHP score were not significantly different between the subgroups of patients with and without evidence of CAD.

Fourteen of 26 living patients underwent maximal exercise testing within 1 year of the current evaluation. The mean age of this subset was 41.8 years. The results are shown in Table V. Patients with heart failure or poor overall medical condition tended to refuse the test and thus the results are biased on the high side. One test was stopped because of electrocardiographic evidence of ischemia and the remainder were patient-terminated because of fatigue. 


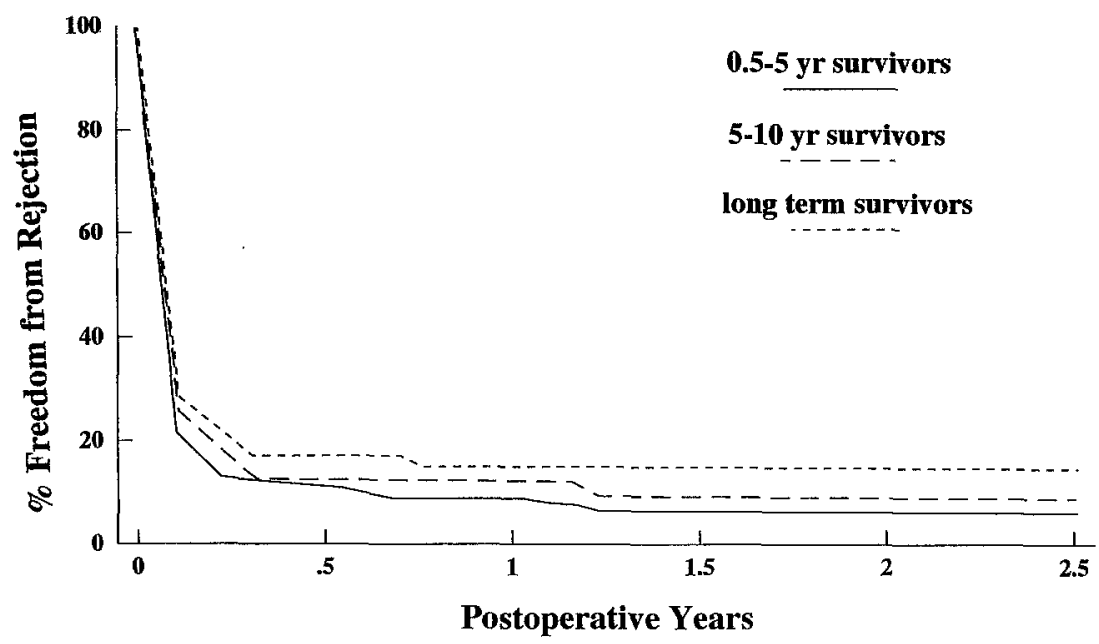

Fig. 1. Kaplan-Meier actuarial freedom from acute rejection episodes for long-term survivors versus less than 5-year survivors, and 5- to 10-year survivors.

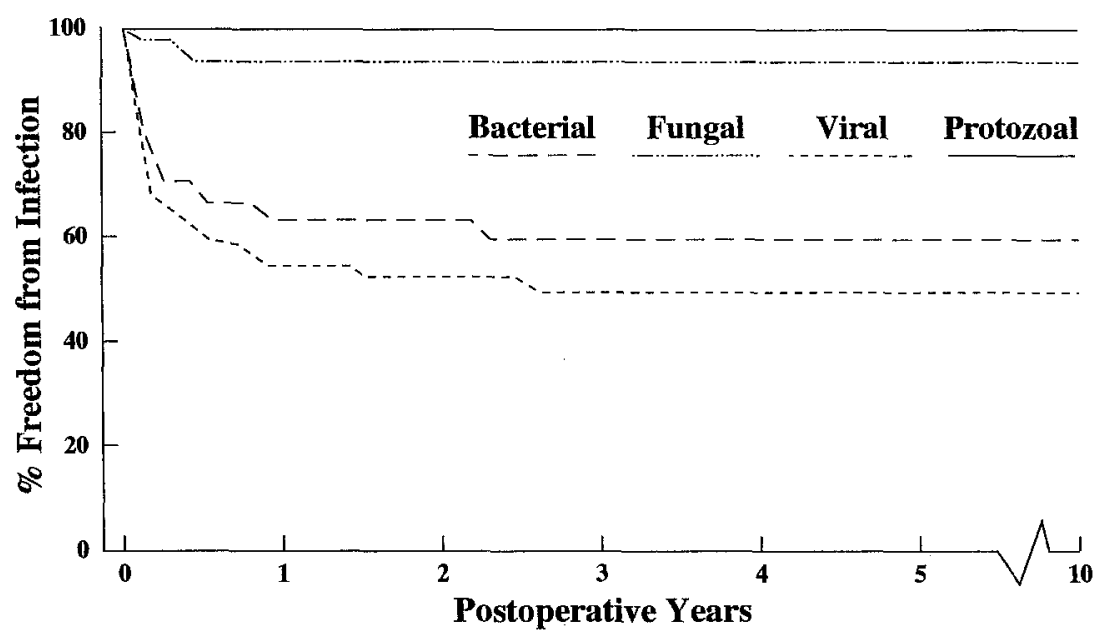

Fig. 2. Kaplan-Meier actuarial freedom from bacterial, fungal, viral, and protozoal infections for long-term survivors.

There was a significant correlation between METS performed and cardiac index on most recent cardiac catheterization (Spearman coefficient 0.81, $p=$ 0.02 ).

Twenty-seven patients completed the GWB and NHP examinations. The average GWB score was 75.3 \pm 21.6 , which is nearly identical to the male normal population mean of $75.1 \pm 14.8(n=79$, mean age 19.6 years). Because scores for part I of the NHP examination could only be properly compared with the use of nonparametric statistics (ranks), a formal analysis could not be done here. The trends did seem to point to higher scores for the patients undergoing transplantation, especially in the oldest age group (50 to 64 years) and subdivisions of mobility, pain, sleep quality, and energy level. NHP scores correlated well with GWB scores (Pearson $R=-0.79$ ). We could not find significant correlations between the GWB or NHP subdivision scores and variables such as number of current medical problems, complications of corticosteroids, or performance on the exercise test. Twenty percent of patients currently smoke, $52 \%$ are currently employed, $73 \%$ have changed jobs since transplantation, $37 \%$ have had a change in marital status since transplantation, and $38 \%$ report some sexual dysfunction.

The actuarial survival curve for the group is shown in Fig. 4, together with the analogous curve 
Table III. Most recent laboratory values $(n=30)$

\begin{tabular}{lrcc}
\hline \multicolumn{1}{c}{ Item } & Value & SD & Range \\
\hline Hematocrit & 42.0 & 4.0 & $34-48$ \\
Glucose $(\mathrm{mg} / \mathrm{dl})$ & 109.7 & 44.4 & $59-257$ \\
Urea nitrogen $(\mathrm{mg} / \mathrm{dl})$ & 23.8 & 12.3 & $6-51$ \\
Creatinine $(\mathrm{mg} / \mathrm{dl})$ & 1.4 & 0.54 & $0.6-2.8$ \\
Total cholesterol $(\mathrm{mg} / \mathrm{dl})$ & 224.2 & 68.9 & $109-445$ \\
Total triglycerides $(\mathrm{mg} / \mathrm{dl})$ & 173.4 & 124.8 & $58-468$ \\
\hline
\end{tabular}

$S D$, Standard deviation.

for all patients who received transplantation at this institution (evaluated on March 1, 1994). The causes of death for the 14 patients who died are displayed in Fig. 5, along with those of patients in the comparison group who survived 0.5 to 5 years. These data include the three patients undergoing retransplantation who died. The predominant cause of death was CAD in the long-term survivors, with no deaths attributable to complications of acute graft rejection. In the short-term survivors, rejection and infection play a dominant role in mortality.

Results of the multivariate analysis of survival are listed in Table VI. The four variables found to be independently correlated with death after transplantation were age at transplantation, preoperative duration of illness, evidence of postoperative CMV infection, and ejection fraction at 12 months after operation. The relative risk of death associated with an increase of 5 years in the duration of illness was $1.53(1.21,1.93)$. An increase of 5 years in the age at transplantation carried a relative risk of 1.28 (1.10, 1.48). A decrease in the ejection fraction at 12 months of 0.1 resulted in a relative risk of death of $1.51(1.10,2.06)$. History of postoperative CMV infection approximately doubled the risk of death $(1.07,3.96)$. As an independent test of the risk associated with CMV infection, life-table calculations using the product limit estimator on the set of all patients undergoing transplantation at this institution were stratified by presence or absence of evidence of postoperative CMV infection. The probability of death within 6 years after operation was $26.7 \%$ without CMV infection and $46.0 \%$ with CMV infection, a relative risk of $1.72(1.20,2.24)$.

\section{Discussion}

Although justification of cardiac transplantation as an effective treatment for end-stage heart failure is provided by prolonged ( $>2$ year) survival and improved functional status, the argument in its favor is greatly strengthened by demonstration of the
Table IV. Most recent cardiac catheterization data $(n=26)$

\begin{tabular}{lrcc}
\hline \multicolumn{1}{c}{ Item } & Mean & SD & Range \\
\hline Pressures (mm Hg) & & & \\
Aortic systolic & 124.8 & 19.5 & $93-175$ \\
Aortic diastolic & 85.2 & 13.3 & $62-115$ \\
RA & 5.5 & 2.0 & $2-9$ \\
PAM & 16.1 & 7.4 & $9-37$ \\
PAW & 9.9 & 5.6 & $3-28$ \\
LVED & 12.8 & 6.1 & $5-25$ \\
Heart rate (beats/min) & 86.4 & 12.0 & $67-102$ \\
CI (L/min per square & 2.9 & 0.57 & $2.1-4.0$ \\
meter) & & & \\
\hline
\end{tabular}

$S D$, Standard deviation; $R A$, right atrial, $P A M$, pulmonary arterial mean; $P A W$, pulmonary arterial wedge; $L V E D P$, left ventricular end-diastolic; $C I$, cardiac index.

possibility of longer-term survival with sustained acceptable functional status. This demonstration was precisely how the current importance of renal transplantation was established 15 years ago. It is appropriate, therefore, to discuss our results in the context of this paradigm.

General medical condition. Aside from rejection and graft $\mathrm{CAD}$, most of the aforementioned medical problems seen in our transplant recipients are probably complications of immunosuppressive therapy. Divakar and colleagues ${ }^{9}$ found a similar variety and incidence of problems in a group of 36 patients surviving greater than 10 years after renal transplantation. The specific role that corticosteroid usage plays in these problems has led many programs to use corticosteroid-free maintenance immunosuppression. Initial results of this regimen are encouraging, ${ }^{10,11}$ although the regimen is not feasible in many patients. We found that the incidence of chronic renal insufficiency was highly correlated to the use of cyclosporine in our group. This problem still remains, although trials using "low-dose" cyclosporine $^{12}$ and cyclosporine analogs such as FK 506 may hold some promise. The incidence of infection in our long-term group was remarkably low relative to that in the two comparison groups, an observation consistent with the fact that infection is a major cause of early death with immunosuppressive therapy. The fact that the curves of actuarial freedom from acute rejection in this group and both comparison groups were all nearly identical means, however, that other factors influenced the group's susceptibility to early fatal infection, such as differences in severity or frequency of rejection or exposure to pathogens, degree of augmentation of immunosuppressive drugs, or individual differences in the de- 


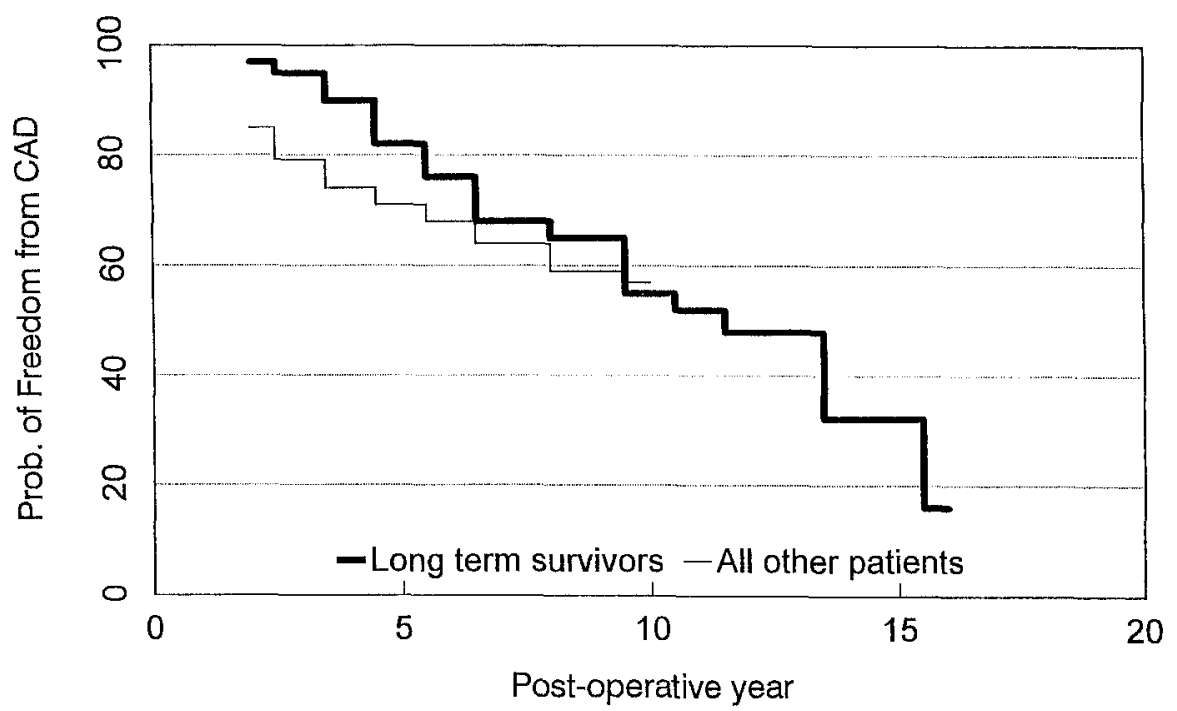

Fig. 3. Kaplan-Meier actuarial freedom from graft CAD for long-term survivors versus all other patients who received transplantation at our institution, updated to January 1994. Prob., Probability.

gree to which given doses of immunosuppressive drugs influence the host response to pathogens.

Graft function. The present work demonstrates, for the first time, preserved graft function well into the second decade after transplantation. Von Scheidt and associates ${ }^{13}$ found no evidence of systolic dysfunction or dilated or restrictive cardiomyopathy in 71 patients followed up to 7 years after operation. Tischler and associates ${ }^{14}$ demonstrated mild increases in left ventricular end-systolic and end-diastolic volumes, but no significant change in left ventricular mass or ejection fraction in 22 patients doing well and followed up a mean of 4 years after operation. Frist and colleagues, ${ }^{15}$ in a study of 174 patients with a mean age of 36 years and follow-up period of 5 years, found no deterioration in graft hemodynamics. Mean cardiac index and left ventricular end-diastolic pressure were almost identical to those in the present group.

Exercise tolerance. Although the exercise tests described in this paper were not standardized to a particular protocol, certain points can be made. First, maximum heart rate and systolic blood pressure were lower than those of a closely age-matched sample of the normal population $(p<0.001){ }^{16}$ but were similar to those of an age-matched group of transplant recipients evaluated a mean of 7 months after operation in a study by Kavanagh and associates. ${ }^{17}$ These results may be explained by the failure of the graft to regain sympathetic innervation a mean of 12 years after implantation. Second, when each patient's estimated maximal oxygen consump-
Table V. Exercise test results $(n=14)$

\begin{tabular}{lrcc}
\multicolumn{1}{c}{ Item } & Mean & \multicolumn{1}{c}{ SD } & Range \\
\hline Duration (min) & 8.7 & 3.5 & $2.0-16.3$ \\
Max. HR (beats/min) & 136.0 & 22.0 & $86-155$ \\
Percent max. HR & 77.3 & 11.3 & $50-92$ \\
Max. systolic BP (mm Hg) & 171.4 & 22.7 & $140-208$ \\
METS & 9.2 & 2.34 & $5.5-12.9$ \\
\hline
\end{tabular}

$S D$, Standard deviation; max, maximum; $H R$, heart rate; $B P$, Blood pressure.

${ }^{*}$ Maximum heart rate $=220-$ age in years.

tion (expressed in METS) is compared with the mean value from an age-matched group of normal subjects not having transplantation, the group performed an average of $1.19 \pm 0.60\left(\mathrm{SEM}^{*}\right)$ METS less $(p=0.08)$ than normal subjects as determined by Bruce, Kusumi, and Hosmer ${ }^{18}$ and $2.55 \pm 0.65$ (SEM) METS less $(p=0.002)$ than normal subjects as published more recently by Morris and colleagues. ${ }^{19}$ These results correspond to an exercise capacity of $88 \% \pm 6 \%$ and $79 \% \pm 6 \%$ of normal, respectively. Yet the group's mean performance was substantially better than the average of $6.2 \pm 1.3$ METS performed in the group of transplant recipients at 7 months after operation in the report of Kavanagh and associates. ${ }^{17}$ A possible interpretation of these data is that exercise capacity improves with duration after operation, which is a hypothesis that could be better tested by a longitudinal study. Alternatively, the data may indicate a time-related

${ }^{*}$ Standard error of the mean. 


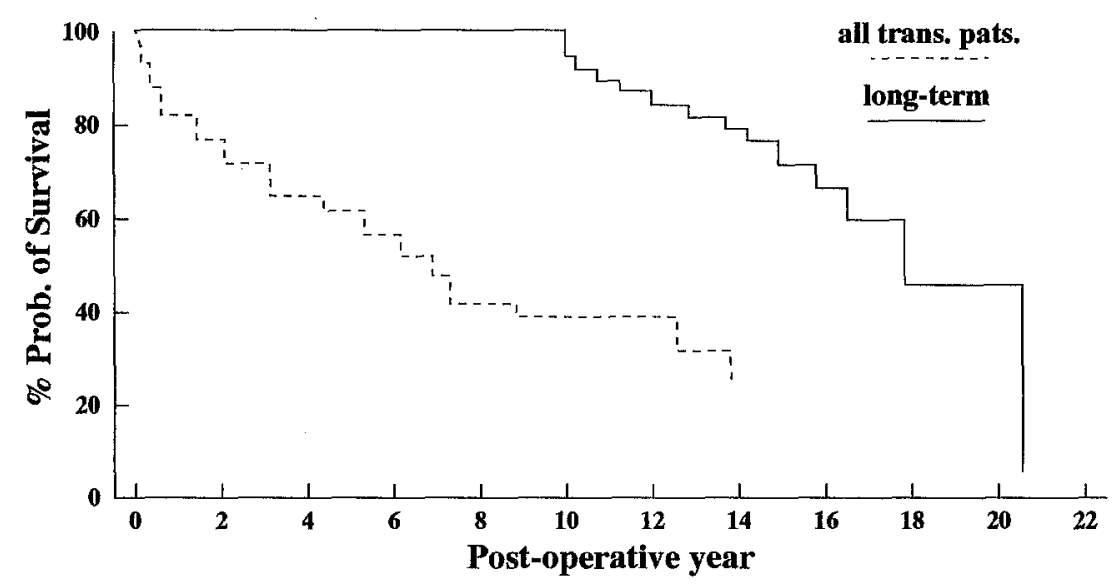

Fig. 4. Kaplan-Meier actuarial probability (Prob.) of survival for long-term survivors (solid curve) and for all patients who recieved transplantation at our institution (all trans. pats.; dashed curve), updated to March 1994.

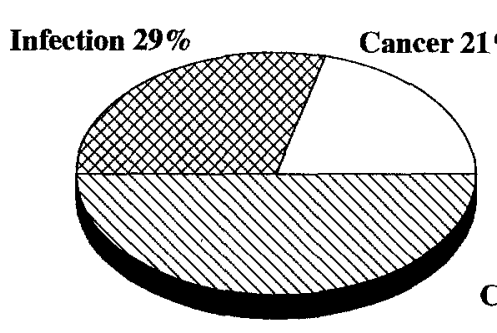

CAD 50\%

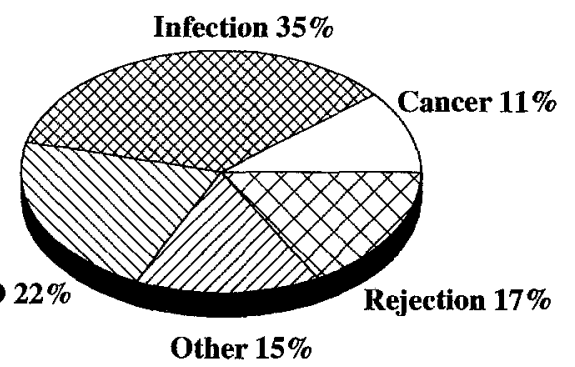

\section{Survival $>10$ yrs \\ $\mathrm{n}=14$}

Survival .5-5 yrs

$$
\mathbf{n}=119
$$

Fig. 5. Distribution of causes of death for long-term survivors compared with that of transplant patients surviving only up to 5 years after transplantation.

bias in patient selection. The cause of the remaining decrement in exercise capacity at 12 years after operation is uncertain, ${ }^{20}$ but it may be compensated for, as Kavanagh and associates ${ }^{17}$ demonstrated, by an exercise training program.

Graft CAD. The most remarkable finding from the angiographic data is that this group of long-term survivors does not escape graft CAD. In fact, the actuarial incidence during the first 10 years after operation is similar to that of the transplant population as a whole. Furthermore, the data indicate that by 20 years after operation essentially all patients can be expected to have graft CAD identifiable by conventional angiographic techniques. The comparison between our group and the overall transplant population is made with some reserva- tion; that is, because the overall transplant population is right-censored by death in the first 10 years, its actuarial prevalence of CAD will be underestimated relative to the estimate for our group. Studies that have attempted to determine risk factors for development of graft $\mathrm{CAD}$ give inconsistent results, ${ }^{21-29}$ and the reported prevalence of CAD varies widely. ${ }^{27,30-32}$ Indeed, we were unable to associate any variables with the presence of CAD in our small group of patients.

Psychosocial evaluation and perceived quality of life. In previous, shorter-term studies in this category, the principal findings were (1) overall sense of well-being improves after transplantation relative to the preoperative state, ${ }^{33-35}$ (2) on the average, there is no deterioration of quality of life with time, $33,35,36$ 
(3) perceived quality does not correlate significantly with medical or demographic data, ${ }^{33}$ (4) a substantial minority of patients have problems that decrease quality of life, especially weakness, sleep disturbances, and sexual dysfunction (impotence), ${ }^{36-40}$ (5) the preoperative perceived quality of life affects the postoperative quality of life and perhaps survival, and (6) about $50 \%$ regain employment after operation.

Several of these findings seem to hold for the present group of patients evaluated more than 10 years after transplantation. The trends in the NHP scores are in accord with those of a large study by Rosenblum and associates, ${ }^{38}$ who found that a group of transplant recipients with a median age of 53 years had Sickness Impact Profile scores "similar to those of cardiac arrest survivors and postmyocardial infarct patients." On the other hand, Aravot and colleagues ${ }^{41}$ found much lower NHP scores at a mean of 22 months of follow-up in a group of patients undergoing transplantation after age 60 years. It could be that a combination of older age and longer postoperative duration correlate with an increasing perception of distress. The percentage of patients employed is similar to that in other reports, indicating that there does not appear to be a decline of employment with duration after operation. As in prior reports, ${ }^{6}$ we could find no correlation between medical data and perceived quality of life. In the future, longitudinal studies (such as those done by Jones $^{33}$ and Caine ${ }^{35}$ and their associates) will permit a better assessment of the long-term, time-dependent trends in these psychosocial and quality-of-life parameters.

Multivariate analysis of survival. Age at the time of transplantation has been cited previously as a correlate with survival duration. ${ }^{1}$ This result should be interpreted with caution, however, because patients who received transplant earlier in our experience were younger at the time of transplantation than were patients who received transplant later in our experience. This bias means that relatively more right-censoring exists in the survival function of the older patients. A second problem with the interpretation of the age factor is that the instantaneous death rate of patients undergoing transplantation at older ages ( $>50$ years old) is significantly influenced by that of the age-matched nontransplanted population. This means that death beyond this age may well not be related to the sequelae of heart transplantation.

The duration of illness before transplantation was an intriguing correlate of survival. Evans and asso-
Table VI. Cox proportional hazards analysis of survival

\begin{tabular}{|c|c|c|c|c|}
\hline & & $\begin{array}{l}\text { Normalized } \\
\text { coefficient }\end{array}$ & $S D$ & $\begin{array}{c}t \\
\text { Statistic }\end{array}$ \\
\hline \multicolumn{5}{|l|}{ Variable } \\
\hline TXAGE & & 0.049 & 0.015 & 3.26 \\
\hline DURILL & & 0.085 & 0.024 & 3.57 \\
\hline CMVINF & & 0.721 & 0.334 & 2.16 \\
\hline $\mathrm{EF} 12$ & & -0.041 & 0.016 & -2.62 \\
\hline \multirow{2}{*}{\multicolumn{5}{|c|}{$\begin{array}{l}\text { Log-likelihood }=-169.9 \\
\text { Multivariate } p=0.0005 \\
n=122\end{array}$}} \\
\hline & & & & \\
\hline & $T X A G E$ & DURILL & $C M V I N F$ & $F \quad E F 12$ \\
\hline \multicolumn{5}{|c|}{ Correlation matrix } \\
\hline TXAGE & 1.0 & & & \\
\hline DURILL & 0.08 & 1.0 & & \\
\hline CMVINF & 0.08 & -0.11 & 1.0 & \\
\hline EF12 & 0.14 & 0.02 & -0.09 & 1.0 \\
\hline
\end{tabular}

$S D$, Standard deviation; TXAGE, age at transplantation; DURILL, preoperative duration of illness; CMVINF, evidence of postoperative CMV infection; $E F 12$, ejection fraction 12 months after operation.

ciates $^{42}$ found that higher levels of pretransplantation functional impairment, as measured by the Karnofsky index, correlated strongly with poorer posttransplantation survival. O'Brien, Buxton, and Ferguson ${ }^{6}$ found that patients indicating fewer quality-of-life restrictions before transplantation had a better likelihood of long-term survival. The degree to which preoperative duration of illness correlates with these two factors is unclear. However, a relationship is suggested by the fact that congestive heart failure is usually progressively debilitating. It could be that subtle aspects of debilitation resulting from cardiac failure are not reversed with cardiac transplantation or are perhaps worsened by the adverse effects of immunosuppressive therapy.

In a recent study by Elkins and associates, ${ }^{43}$ primary or recurrent $\mathrm{CMV}$ infection occurred in $64 \%$ of heart transplant recipients a mean of 164 and median of 84 days after operation. Some of the decrease in survival as a result of CMV infection can be accounted for by various manifestations of the disease or syndrome. The correlation of a history of CMV infection with survival and with CAD incidence was first noted by Grattan and colleagues ${ }^{44}$ in 1989 . CMV can regulate major histocompatibility complex class I expression on smooth muscle cells, ${ }^{45}$ and CMV infection is associated with progressive intimal thickening of intramyocardial arterioles in cardiac grafts. ${ }^{46} \mathrm{It}$ is thus likely that the late effects of CMV infection 
on survival are due to its contribution to graft CAD. This notion is supported by our multivariate analysis, that is, partly because graft CAD is not usually detectable in the first 12 to 24 months by conventional angiographic analysis, CMV infection, and not graft CAD per se, was an independent risk factor for mortality.

The ejection fraction at 12 months was the fourth significant correlate with survival in our analysis. This result is not surprising. Myocardial dysfunction can result from a variety of causes, such as severe recurrent rejection, early graft $\mathrm{CAD}$, myocarditis (often viral or drug-induced), cardiomyopathy, or "nonspecific" graft failure. In our analysis, ejection fraction at 12 months correlated somewhat with detection of CAD at 12 months (Pearson coefficient $=-0.35$ ), but not with diastolic blood pressure at 12 months, the total number of episodes of acute rejection, or the incidence of all viral infections.

With respect to the aforementioned multivariate analysis, it is important to note the limitations of the proportional hazards method. Although it is relatively easy to apply, it assumes that all covariates will have the same shape of hazard function. A more versatile approach would determine each hazard function, thus perhaps allowing some understanding of the biologic nature of the dependence of the covariates in the model.

The vast majority of this group of patients underwent operation in an era when many of the improved methods of patient management now in use were unavailable, such as cyclosporine, "steroidfree" maintenance immunosuppression, specific anti-T cell induction immunosuppression, routine early antibiotic prophylaxis against CMV (ganciclovir) and Pneumocystis carinii (trimethoprim/sulfamethoxazole), third-generation antibiotics, early use of prophylactic calcium channel blockade (diltiazem) for reduction of graft CAD progression, ${ }^{47}$ improved donor selection, and other empiric features gained from acceleration of experience in cardiac transplantation worldwide after about 1983. Thus the conclusions we have drawn from our study are likely to be unique to this group.

Conclusions. Results of this study of long-term survivors of heart transplantation support the following conclusions: (1) graft function is well-preserved into the second decade after transplantation, (2) exercise capacity is $80 \%$ to $90 \%$ of that of the age-matched general population, (3) patient-perceived health and well-being (by NHP and GWB examinations) is close to that of the age- and sex-matched general population, (4) the cumulative incidence of graft CAD is similar to that of the cardiac transplant population as a whole, continues to develop after 10 years, and is a significant source of mortality, and (5) long-term survival is correlated with four factors that can be evaluated by 1 year after transplantation; younger age at time of transplantation, shorter preoperative duration of illness, absence of postoperative CMV infection, and larger left ventricular ejection fraction at 1 year after operation.

Cardiac transplantation can provide patients with end-stage cardiac failure an acceptable general medical condition, functional status, and perceived quality of life well into the second decade after operation.

\section{REFERENCES}

1. Grattan MT, Moreno-Cabral CE, Starnes VA, Oyer PE, Stinson EB, Shumway NE. Eight-year results of cyclosporine-treated patients with cardiac transplants. J THORAC CARDIOVASC SuRg 1990;99:500-9.

2. Hunt SM, McKenna SP, McEwen J, Williams J, Papp E. The Nottingham Health Profile: subjective health status and medical consultations. Soc Sci Med 1981; 15A:221-9.

3. Hunt SM, McKenna SP. The Nottingham Health Profile user's manual. Revised edition. Manchester: Southern Hey, 1991.

4. McKenna SP, Hunt SM, McEwen J. Weighting the seriousness of perceived health problems using Thurstone's method of paired comparisons. Int J Epidemiol 1981;10:93-7.

5. Fazio AF. A concurrent validational study of the NCHS general well-being schedule. Hyattsville, Maryland: National Center for Health Statistics, 1989; HEW publication no. (HRA) 78-1347. (Vital and health statistics; series 2; no 73).

6. O'Brien BJ, Buxton MJ, Ferguson BA. Measuring the effectiveness of heart transplant programmes: quality of life data and their relationship to survival analysis. J Chronic Dis 1987;40(suppl 1):137S-58S.

7. Kalbfleisch J, Printice R. The statistical analysis of failure time data. New York: John Wiley and Sons, 1980:46-50.

8. Lee ET. Statistical methods for survival data analysis. 2nd ed. New York: John Wiley and Sons, 1992:243-80.

9. Divakar D, Bailey RR, Lynn KL, Robson RA. Long term complications following renal transplantation. N Z Med J 1991;104:352-4.

10. Pritzker MR, Lake KD, Reutzel TJ, et al. Steroid-free maintenance immunotherapy: Minneapolis Heart Institute experience. J Heart Lung Transplant 1992;11: $415-20$. 
11. O'Connell JB, Bristow MR, Rasmussen LG, et al. Cardiac allograft function with corticosteroid-free maintenance immunosuppression. Circulation 1990; 82(Suppl):IV318-21.

12. Gonwa TA, Mai ML, Pilcher J, et al. Stability of long-term renal function in heart transplant patients treated with induction therapy and low-dose cyclosporine. J Heart Lung Transplant 1992;11:926-8.

13. von Scheidt W, Ziegle U, Kemkes BM, Reichart B, Erdminn E. Long-term myocardial function after heart transplantation. Thorac Cardiovasc Surg 1993; 41:156-62.

14. Tischler MD, Lee RT, Plappert T, Mudge GH, St. John Sutton M, Parker JD. Serial assessment of left ventricular function and mass after orthotopic heart transplantation: a 4-year longitudinal study. J Am Coll Cardiol 1992;19:60-6.

15. Frist WH, Stinson EB, Oyer PE, Baldwin JC, Shumway NE. Long-term hemodynamic results after cardiac transplantation. J THORAC Cardiovasc Surg 1987;94:685-93.

16. Shephard RJ. Responses of the cardiac transplant patient to exercise and training. Exercise Sport Sci Rev 1992;20:297-320.

17. Kavanagh T, Yacoub MH, Mertens DJ, Kennedy J, Campbell RB, Sawyer P. Cardiorespiratory responses to exercise training after orthotopic cardiac transplantation. Circulation 1988;77:162-71.

18. Bruce RA, Kusumi F, Hosmer D. Maximal oxygen intake and nomographic assessment of functional aerobic impairment in cardiovascular disease. Am Heart J 1973;85:546-62.

19. Morris CK, Myers J, Froelicher VF, Kawaguchi T, Ueshima K, Hideg A. Nomogram based on metabolic equivalents and age for assessing aerobic exercise capacity in men. J Am Coll Cardiol 1993; 22:175-82.

20. Braith RW, Limacher MC, Leggett MS, Pollock ML. Skeletal muscle strength in heart transplant recipients. J Heart Lung Transplant 1993;12:1018-23.

21. Stovin PG, Sharples LD, Schofield PM, et al. Lack of association between endomyocardial evidence of rejection in the first six months and the later development of transplant-related coronary artery disease. J Heart Lung Transplant 1993;12:110-6.

22. Dresdale AR, Kraft PL, Paone G, et al. Reduced incidence and severity of accelerated graft atherosclerosis in cardiac transplant recipients treated with prophylactic antilymphocyte globulin. J Cardiovasc Surg 1992;33:746-53.

23. Carrier M, Pelletier G, Leclerc Y, et al. Accelerated coronary atherosclerosis after cardiac transplantation: major threat to long-term survival. Can Surg 1991;34:133-6.

24. Eich D, Thompson JA, Ko DJ, et al. Hypercholesterolemia in long-term survivors of heart transplanta- tion: an early marker of accelerated coronary artery disease. J Heart Lung Transplant 1991;10:45-9.

25. Advancovic B, Poindexter S, Birovljev N, et al. Risk factors for development of accelerated coronary artery disease in cardiac transplant recipients. Eur J Cardiothorac Surg 1990;4:309-12.

26. McDonald K, Rector TS, Braulin EA, Kubo SH, Olivari MT. Association of coronary artery disease in cardiac transplant recipients with cytomegalovirus infection. Am J Cardiol 1989;64:359-62.

27. Ratkovec RM, Wray RB, Renlund DG, et al. Influence of corticosteroid-free maintenance immunosuppression on allograft coronary artery disease after cardiac transplantation. J Thorac CARdrovasc Surg 1990;100:6-12.

28. Gao SZ, Schroeder JS, Hunt SA, Valantine HA, Hill IR, Stinson EB. Influence of graft rejection on incidence of accelerated graft coronary artery disease: a new approach to analysis. J Heart Lung Transplant 1993;12:1029-35.

29. DeCampli WM, Johnson DE, Gao SZ, et al. Transplant coronary vascular disease: histomorphometric properties and clinical correlations. Curr Surg 1988; 45:477-80.

30. Hunt S, Billingham M. Long-term results of cardiac transplantation. Annu Rev Med 1991;42:437-47.

31. Heublein B, Haverich A, Borst HG. Long-term follow-up after orthotopic heart transplantation. Thorac Cardiovasc Surg 1990;38:285-90.

32. Alibelli-Chemarin MJ, Puel J, Assoun B, Galinier M, Khoury FL, Bounhoure J-P. Comparison of plaque morphology by intravascular ultrasound and clinical presentation in coronary artery disease. In: Abstracts and original contributions of the fortythird meeting of the American College of Cardiology. Journal of the American College of Cardiology. New York: Elsevier Science, Abstract 742-4, 1994:171A.

33. Jones BM, Taylor F, Downs K, Spratt P. Longitudinal study of quality of life and psychological adjustment after cardiac transplantation. Med J Aust 1992;157:24-6.

34. Mai FM, McKenzie FN, Kostuk WJ. Psychosocial adjustment and quality of life following heart transplantation. Can J Psychiatry 1990;35:223-7.

35. Caine N, Sharples LD, English TAH, Wallwork J. Prospective study comparing quality of life before and after heart transplantation. Transplant Proc 1990;22:1437-9.

36. Strauss B, Thormann T, Strenge $H$, et al. Psychosocial, neuropsychological and neurological status in a sample of heart transplant recipients. Qual Life Res 1992;1:119-28.

37. Angermann CE, Bullinger $M$, Spes $\mathrm{CH}$, Zellner $\mathrm{M}$, Kemkes BM, Theisen K. Quality of life in long-term survivors of orthotopic heart transplantation. Zeitschr Kardiol 1992;81:411-7.

38. Rosenblum DS, Rosen ML, Pine ZM, Rosen SH, 
Borg-Stein J. Health status and quality of life following cardiac transplantation. Arch Phys Med Rehabil 1993;74:490-3.

39. Mai FM. Psychiatric aspects of heart transplantation. Br J Psychiatry 1993;163:285-92.

40. Mulligan T, Sheehan H, Hanrahan J. Sexual function after heart transplantation. J Heart Lung Transplant 1991;10:125-8.

41. Aravot DJ, Banner NR, Khaghani A, et al. Cardiac transplantation in the seventh decade of life. Am J Cardiol 1989;63:90-3.

42. Evans RW, Manninen DL, Overcast TD, et al. The national heart transplantation study: final report. Seattle, Washington: Battelle Human Affairs Research Center, 1984.

43. Elkins CC, Frist WH, Dummer JS, et al. Cytomegalovirus disease after heart transplantation: is acyclovir prophylaxis indicated? Ann Thorac Surg 1993;56:1267-73.

44. Grattan MT, Moreno-Cabral CE, Starnes VA, Oyer PE, Stinson EB, Shumway NE. Cytomegalovirus infection is associated with cardiac allograft rejection and atherosclerosis. JAMA 1989;261:3561-6.

45. Hosenpud JD, Chou SW, Wagner CR. Cytomegalovirus-induced regulation of major histocompatibility complex class I antigen expression in human aortic smooth muscle cells. Transplant 1991;52:896-903.

46. Koskinen PK, Nieminen MS, Krogerus LA, et al. Cytomegalovirus infection and accelerated cardiac allograft vasculopathy in human cardiac allografts. J Heart Lung Transplant 1993;12:724-9.

47. Schroeder JS, Gao SZ, Alderman EL, Hunt S, Hill I, Stinson EB. Prevention of transplant accelerated coronary vascular disease with diltiazem. In: Abstracts and original contributions of the forty-third meeting of the American College of Cardiology. Journal of the American College of Cardiology. New York: Elsevier Science, Abstract 758-5, 1994:231A.

\section{Discussion}

Dr. Alfredo Trento (Malibu, Calif.). The importance of this review is not in the comparison between different groups of patients undergoing transplantation. These were groups of patients who received transplantation in different periods of time when there were different immunosuppressive regimens and in which there were very different understandings about the side effects of immunosuppression therapy and about how to prevent the side effects. The conclusions are extremely important and are those that we transplant surgeons have been expecting.

The conclusions are that the quality of the life for the surviving patients is similar to that of an age-matched population and that the main cause of late death beyond 10 years after transplantation is CAD. CAD is progressive to the point that if we wait long enough every transplant recipient would be affected. The other conclusion is that rejection and $C A D$ together are the main cause of death in the patients who died within 10 years of transplantation. This brings me to my first question. In the manuscript the authors reported that only $15 \%$ of the patients are free of rejection 6 months after transplantation. I reviewed our experience and found that at 1 year after transplantation $60 \%$ of the patients are free of rejection and $60 \%$ of them have also been weaned from steroids. Can you clarify this high prevalence of rejection, and do you think that played a role in the long-term survival and in the incidence of CAD?

Dr. DeCampli. I think the important factor here is that these patients received transplantation in a unique interval of time in which we were on a very steep learning curve to understanding how to best manage transplant cases preoperatively, immediately postoperatively, and in the long term. The patients underwent transplantation between 1970 and 1982 . Only a few of them ever received cyclosporine. It was probably the absence of cyclosporine, and not so much the absence of anti-CD4 induction therapy, that accounted for the high incidence of rejection in the first couple of months after operation. I qualify that by saying that even with the addition of cyclosporine in the modern era and with the addition of OKT3 routinely, Stanford's incidence of rejection in the first 6 months is still $75 \%$ to $80 \%$. That number does differ from yours and I cannot, offhand, explain that difference.

Dr. Trento. The manuscript points out the effect of $\mathrm{CMV}$ infection on survival: history of postoperative CMV infection doubles the risk of death at 6 years after transplantation. It is clear now that CMV infection seems to trigger some sort of generalized immunologic reactivation that may trigger a form of acute or chronic rejection that we do not know how to diagnose or to treat. This may play a role in the occurrence of CAD. For this reason, we have started at our institution an aggressive and also costly type of prophylaxis against CMV infection, which involves the administration of hyperimmune gamma globulin weekly for the first 4 weeks and then intravenous administration of ganciclovir for 2 weeks followed by oral acyclovir for 3 months. With this regimen we have decreased the incidence of postoperative CMV infection from a range of about $35 \%$ to $70 \%$ as reported in the literature and from a range of about $50 \%$ reported in this paper to $4 \%$ in patients who were CMV positive before transplantation and to about $26 \%$ in the difficult group of patients who were CMV negative before transplantation and received a CMV-positive organ. Would you comment on this aspect and also basically on the approach that Stanford is taking at the present for CMV prophylaxis?

Dr. DeCampli. Stanford's approach currently is as follows. If I designate $\mathrm{D}$ as donor and $\mathrm{R}$ as recipient and D-negative R-negative means that neither was CMV positive, then we have no prophylaxis for D-negative $\mathrm{R}$-negative situations. In the $\mathrm{D}$-negative $\mathrm{R}$-positive and D-positive R-positive situations, we give ganciclovir for 2 weeks intravenously and for 2 weeks orally for a total of 4 weeks of therapy. With this regimen we have achieved a significant reduction, as compared with the results of these long-term survivors, in CMV infection in the first 6 months. Thus the incidence is now approximately $15 \%$ rather than $50 \%$. In the so-called CMV mismatch group, that is, D-positive R-negative, which is the most difficult group, we have found no benefit with 4 weeks of ganciclovir therapy. Stanford has empirically extended its cov- 
erage from 4 to 6 weeks. Stanford has found no particular benefit to the use of CMV antiglobulin, in particular in the $\mathrm{D}$-positive $\mathrm{R}$-negative group. We have had no early deaths from CMV infection in the past 5 years. However, we cannot yet make a statement on the effect of any of these prophylaxis regimens on the later incidence of graft CAD. I am not sure any institution can do that yet.

Dr. Trento. It is my feeling that any immunosuppression based on cyclosporine or cyclosporine analogs has reached a plateau and basically we have gotten out of cyclosporine everything that cyclosporine can give. Further improvement in results will probably be only minor unless a qualitative change in the immunosuppressive therapy is made. Would you like to comment on this last point?

Dr. DeCampli. If you ask how we can best improve the survival of patients undergoing transplantation and if you make the assumption that graft $C A D$ is caused by a chronic rejection phenomenon, then the answer is more selective immunosuppression leading ultimately to what is now popularly known as "organ-specific clonal anergy." One possible way of achieving this is the use of total lymphoid irradiation for tolerance induction. The manner in which stem cells regenerate after total lymphoid irradiation is in a peculiar order that, in fact, favors acceptance of a transplanted organ; that is to say, suppressor cells seem to recover first after total lymphoid irradiation, blunting the effect of the particular lymphoid subset that acts against the implanted organ. Total lymphoid irradiation, in conjunction with the use of a new anti-CD4 antibody that actually depletes CD4 cells (rather than just altering their surface membrane structure), is currently being investigated as a combination that could give us more tolerance induction and organ-specific clonal anergy. Dr. Bruce Reitz is working on this problem in the laboratory at Stanford currently with primates. 